\title{
Neuralgia trigeminal y algunas consideraciones en la práctica clínica de la odontología
}

Trigeminal neuralgia and some considerations in practice dentistry clinic Neuralgia do trigêmeo e alguns considerações na prática clínica odontológica

\section{Lesbia Rosa Tirado Amador ${ }^{1}$ \\ Lenin Torres Osorio ${ }^{2}$ Jhon Alex Arce Vanegas ${ }^{3}$}

Recibido: 26 de septiembre de 2018 Aprobado: 8 de abril de 2019

Publicado: 1 de julio de 2019

Cómo citar este artículo: Tirado Amador LR, Torres Osorio L, Arce Vanegas JA. Neuralgia trigeminal y algunas consideraciones en la práctica clínica de la odontología. Rev Nac Odontol. 2019;15(29):1-15. doi: https://doi.org/10.16925/2357-4607.2019.02.06

Artículo de Reflexión derivado de Investigación. https://doi.org/10.16925/2357-4607.2019.02.06

1 Universidad del Sinú seccional Cartagena, Cartagena, Colombia

ORCID: https://orcid.org/0000-0003-2821-6762

Lesbia Rosa Tirado Amador, Avenida Pedro de Heredia sector María Auxiliadora, Cartagena de Indias, Colombia.

Correo electrónico: lesbia.tirado@unisinu.edu.co

2 Universidad del Sinú seccional Cartagena, Cartagena, Colombia

ORCID: https://orcid.org/0000-0002-6793-1454

3 Universidad del Sinú seccional Cartagena, Cartagena, Colombia

ORCID: https://orcid.org/0000-0001-8706-5429 


\title{
Resumen
}

Introducción: en la práctica odontológica es frecuente la demanda de odontólogos con competencias para el diagnóstico oportuno e instauración del tratamiento. Algunas condiciones patológicas ocasionalmente no son del interés directo para el odontólogo en su lista de prioridades, sin embargo, todo y cuanto le concierne al sistema estomatognático y a su funcionamiento debe ser considerado relevante, particularmente lo referente al Nervio Trigémino ( $V$ par craneal), encargado de transmitir sensaciones a la boca, dientes, cara, cavidad nasal e inervación de músculos masticadores. Algunas condiciones pueden provocar neuralgia trigeminal, una afección dolorosa e incapacitante, que puede afectar la calidad de vida.

Objetivo: generar interés en el profesional de odontología respecto a neuralgia trigeminal y el rol que puede asumir en la oportuna atención del paciente y prevención durante algunos procedimientos dentales que podrían desencadenarla.

Metodología: en la atención clínica es importante reflexionar acerca de la anatomía del nervio trigémino y respecto a la neuralgia trigeminal, su definición, características, clasificación, etiología, consideraciones diagnósticas, la relación existente entre el odontólogo, la consulta odontológica y los pacientes con neuralgia trigeminal, así como la toma de decisiones en el tratamiento e intervención del odontólogo en esta afección.

Conclusiones: el conocimiento de los factores desencadenantes, signos, síntomas y alternativas terapéuticas permite al odontólogo contribuir para interactuar de manera activa e interdisciplinaria con el equipo de atención, más aún cuando se trata de uno de los principales nervios sobre los cuales se influye durante los tratamientos odontológicos.

Palabras clave: nervio trigémino, neuralgia, neuralgia del trigémino, dolor, odontólogos (DeCS -Bireme).

\begin{abstract}
Purpose: To generate interest in the dental professional regarding trigeminal neuralgia and the role it can assume in the timely care of the patient and its prevention during some dental procedures that could trigger it. Description: In dental practice, the demand of dentists with competence for opportune diagnosis and establishment of treatment is high. Some pathological conditions are occasionally not of direct interest to the dentist in their list of priorities, however, everything and that concerns the stomatognathic system and its operation should be considered relevant, particularly with regard to the Trigeminal Nerve ( $V$ cranial nerve), responsible for transmitting sensations to the mouth, teeth, face, nasal cavity and innervation of chewing muscles. Some conditions can cause Trigeminal Neuralgia, a painful and disabling condition, which can affect quality of life. Points of view: In clinical care, it is important to reflect on the anatomy of the trigeminal nerve and on trigeminal neuralgia, its definition, characteristics, classification, etiology, diagnostic considerations, the relationship between the dentist, the dental consultation and the patients with trigeminal neuralgia, as well as decision-making in the treatment and intervention of the dentist for this condition. Conclusions: Knowledge of the triggering factors, signs, symptoms and therapeutic alternatives allow the dentist to contribute to interacting in an active and interdisciplinary way with the care team; even more so when it is one of the main nerves that can be influenced during dental treatments.
\end{abstract}

Keywords: Trigeminal Nerve, Neuralgia, Trigeminal Neuralgia, Pain, Dentist (MeSH-Database). 


\section{Resumo}

Introdução: na prática odontológica, há uma demanda frequente de dentistas com competências para o diagnóstico oportuno e a introdução do tratamento. Algumas condições patológicas ocasionalmente não são de interesse direto do dentista em sua lista de prioridades, no entanto, tudo o que diz respeito ao sistema estomatognático e seu funcionamento deve ser considerado relevante, principalmente no que diz respeito ao Nervo Trigêmeo (nervo craniano $V$ ), responsável transmitir sensações à boca, dentes, face, cavidade nasal e inervação dos músculos mastigatórios. Algumas condições podem causar neuralgia do trigêmeo, uma condição dolorosa e incapacitante, que pode afetar a qualidade de vida.

Objetivo: gerar interesse no profissional odontológico em relação à neuralgia do trigêmeo e o papel que ele pode assumir no atendimento oportuno do paciente e na prevenção durante alguns procedimentos odontológicos que possam desencadear esse problema.

Metodologia: no atendimento clínico, é importante refletir sobre a anatomia do nervo trigêmeo e a neuralgia do trigêmeo, sua definição, características, classificação, etiologia, considerações diagnósticas, a relação entre o dentista, o consultório odontológico e os pacientes com neuralgia. trigeminal, bem como tomada de decisão no tratamento e intervenção do dentista nessa condição.

Conclusões: o conhecimento dos gatilhos, sinais, sintomas e alternativas terapêuticas permite ao dentista contribuir para interagir ativa e interdisciplinarmente com a equipe de atendimento, ainda mais quando é um dos principais nervos influenciados durante tratamentos dentários.

Palavras-chave: nervo trigêmeo, neuralgia, neuralgia do trigêmeo, dor, dentista (DeCS-Bireme).

\section{Introducción}

El odontólogo es un profesional del área de la medicina que se encarga de la salud bucal de sus pacientes y además asume el compromiso de propiciar el bienestar al sistema estomatognático; sin embargo, en ocasiones, existen ciertas situaciones en las que la responsabilidad del manejo terapéutico no es exclusiva de su área y se requiere de un trabajo colaborativo en equipo. Respecto a esto último, se pueden considerar algunas neuralgias, en específico la neuralgia trigeminal (NT), que afecta al nervio trigémino ( $\mathrm{V}$ par craneal). Este nervio es de relevancia clínica para el odontólogo pues es el encargado de la transmisión de sensaciones a la boca, los dientes, la cara y la cavidad nasal e inervación de los músculos masticadores; además, sobre sus ramas distales, el odontólogo aplica ciertos anestésicos para lograr la ejecución de múltiples procedimientos. Por lo que es necesario disponer de ciertos lineamientos teóricos básicos respecto a la distribución de este nervio, las características del dolor en caso de NT, la identificación de situaciones que pueden inducir NT y su etiología (aspecto muy diverso), algunas consideraciones clínicas para su diagnóstico y ayudas complementarias así como la relación existente entre el odontólogo, la consulta odontológica y los pacientes con NT respecto a ¿qué sucede en el diagnóstico de esta alteración al interior de la consulta odontológica? y ¿cuáles son los tratamientos 
disponibles y la posible participación del odontólogo en la prescripción de fármacos en caso de NT? Todo esto influye en que el odontólogo como parte del equipo interdisciplinario de atención en salud, pueda contribuir en el diagnóstico y tratamiento oportuno y eficaz, ya sea aplicando su conocimiento para su tratamiento quizás en lo que respecta al manejo farmacológico u orientando al paciente y derivando con los profesionales involucrados en la atención del dolor tales como médicos de atención primaria, neurólogos o especialistas en dolor.

\section{Descripción del nervio trigémino}

Este aspecto puede ser ocasionalmente un dolor de cabeza para estudiantes y profesional del área de la odontología, pues el $\mathrm{V}$ par craneal posee un amplio recorrido o distribución, lo que muchas veces es omitido o subvalorado; sin embargo es importante recordar que es un nervio con función mixta con componentes sensitivos y motores, los cuales se encuentran distribuidos a través de 3 segmentos: 1) segmento intra-axial (constituido por 4 núcleos que son: núcleo mesencefálico que se encarga de la propiocepción facial proveniente de los dientes, el paladar duro y la articulación temporomandibular; núcleo sensorial principal que conduce la sensación táctil a la cara; núcleo espinal trigeminal que contribuye a la percepción del dolor y la temperatura y núcleo motor trigeminal que inerva los músculos de la masticación, así como al tensor del paladar y del tímpano, el milohioideo y el vientre anterior del digástrico); 2) segmento cisternal (que por medio de dos raíces, una sensitiva que es la de mayor tamaño y una raíz más pequeña que es motora atraviesa la cisterna prepontina y entra al cavum de Meckel a través del "porus trigeminus"); y 3) segmento interdural (a nivel del cual la rama sensitiva forma una estructura llamada ganglio de Gasser que se posiciona en el aspecto inferior del cavum y da origen a tres ramas: $\vee 3$ mandibular (se divide en dos ramas motoras y tres sensitivas), V2 maxilar (con distribución para inervar mejillas y dientes superiores, a nivel del piso orbitario como nervio infraorbitario y una porción pterigopalatina de la que derivan ramas como el nervio alveolar posterosuperior, nervio cigomático y dos ramas pterigopalatinas) y $\vee 1$ oftálmica (ramificada en nervio lagrimal, nervio frontal y nervio nasociliar) (1). Esta distribución muchas veces parece que debe ser competencia del médico y muchos estudiantes y profesionales de la odontología sólo suelen recordar los agujeros por donde emergen las ramas terminales hacia la cavidad bucal, pero deberán asumir una actitud de compromiso donde comprendan que del reconocimiento de la distribución anatómica depende 
una adecuada valoración del compromiso que pueda tener este nervio, pues la localización de las áreas afectadas puede orientar para identificar la rama que se encuentra comprometida. Lo que obliga a la comunidad odontológica a retomar los tratados de anatomía y muy seguramente el módulo de sistema nervioso, volverlos parte de su biblioteca personal y de su material de consulta diario, pues el verdadero clínico de la estomatología no puede aislar el sistema nervioso de las estructuras del sistema estomatognático.

\section{Neuralgia del trigémino (NT) y sus características}

La anatomía y distribución del nervio trigémino es imperativa para el estudiante de odontología en formación y para el profesional en su práctica cotidiana, es importante recordar que esta condición ha sido definida como un trastorno neuropático del quinto par craneal y que su importancia radica principalmente en la severidad que posee y en la alta frecuencia con la que se presenta, incluso se ha reportado una prevalencia de 155 casos por cada 1000000 habitantes (2). Por lo anterior, existe la probabilidad de que se presente alguna vez en el servicio de odontología algún caso de NT y lo que se recomienda para la identificación de este trastorno en el nervio trigémino es tener muy presente algunas características tales como encontrarse localizado en una o varias ramas del V par craneal (lo que nuevamente direcciona al conocimiento de su distribución), poseer intensidad alta (considerar la sintomatología referida por el paciente y la repercusión que tiene el dolor en actividades cotidianas de su vida), tener duración de segundos a minutos, posible recurrencia de los episodios con periodos libres de dolor (3) y ser desencadenado por algunos estímulos (térmicos y táctiles), actividades vitales (la masticación, hablar), actividades cotidianas (lavarse los dientes, la aplicación de cosméticos faciales, sonreír, bostezar) (4). Lo anterior resultará crucial al momento de tomar decisiones terapéuticas o de considerar la remisión hacia otro miembro del equipo de atención en salud pues la omisión de estas características puede ser un factor que coloque en riesgo el bienestar del paciente, a causa de un diagnóstico incorrecto que obligaría a someter al paciente a tratamientos innecesarios y por ende al retardo en la instauración oportuna del tratamiento idóneo. 


\section{Respecto a la clasificación de la NT}

Se recomienda realizar la diferenciación entre NT idiopática, clásica y secundaria, comprendiendo la importancia y lo indispensable del uso de imagenología para su diagnóstico. En la neuralgia trigeminal idiopática no hay contacto neurovascular o cambios en la raíz del nervio trigémino, en la neuralgia trigeminal clásica se puede identificar la presencia de compresión neurovascular (comúnmente la arteria cerebelosa superior) con la presencia de cambios morfológicos en la raíz del trigémino empleando resonancia magnética. En tanto, la neuralgia secundaria es consecuencia de una enfermedad neurológica subyacente identificable como los tumores de ángulo cerebelopontino o esclerosis múltiple $(5,6)$. Este aspecto es un tópico importante a considerar, pues para el diagnóstico adecuado es importante reconocer el origen de la neuralgia trigeminal, y para esto es imprescindible hacer uso de todas las herramientas clínicas disponibles y, en el particular caso del uso de imagenología, obliga a pensar en la necesidad de comprometerse con la adquisición de conocimientos básicos en radiología, para saber interpretar la anatomía patológica, que se convierte en una necesidad y competencia a desarrollar por parte de los profesionales de la atención en salud.

\section{Estado del arte respecto a factores etiológicos en NT}

En referencia a este aspecto de tanta relevancia, es menester recordar que de la identificación del agente causal o etiológico depende la instauración del mejor tratamiento ante cualquier patología. De esta manera, una adecuada historia clínica, producto de la valoración clínica del profesional y que se complementa con la información que deriva de una anamnesis confiable, puede orientar al clínico hacia la posible verdadera causa de la NT, una entidad para la cual existe una diversidad de factores etiológicos relacionados. Algunos factores son los de tipo hereditario, para este aspecto se menciona un patrón de herencia autosómico dominante, responsable de la neuralgia trigeminal clásica familiar (NTCF), en la cual existen algunos factores genéticos determinantes tales como inestabilidad neuronal, anomalías en la morfología de la base del cráneo y la predisposición a desarrollar arterioesclerosis prematura $(7,8)$. En otros casos, se ha optado por considerar algunos factores asociados a enfermedades de tipo sistémicos, tal es el caso de la NT que puede surgir después de la infección por virus del herpes, presencia de esclerosis múltiple, presencia de diabetes o enfermedad reumática (9). Otra posible etiología es la que concierne al propio del nervio trigémino en la cual puede considerarse su aparición como respuesta a un proceso oncológico (presencia de un 
tumor), consecuencia de un trauma, por crisis de migraña recurrente, dolor central, presencia de enfermedades inflamatorias odontogénicas, patología otolaringológica o la presencia de malformación de la arteria venosa, schwannomas vestibulares, meningiomas, quistes epidermoides, tuberculomas, aneurismas, agregación y oclusión vascular después de aracnoiditis además de un posible origen polietiológico en el que se incluyen todos los factores que pueden afectar el nervio trigémino y que provocan desmielinización y distrofia (10).

\section{Consideraciones para el diagnóstico en NT}

Para el diagnóstico de NT es importante siempre reconocer que debe ser el resultado de articular el uso de herramientas clínicas e imagenológicas. Referente a las consideraciones clínicas, se sugiere tener en cuenta la presencia de al menos cuatro de los nueve criterios clínicos, que han sido previamente descritos por la International Headache Society (IHS) (4) (contenidos en la tabla 1) y que son muy prácticos para su implementación. Por otro lado, las ayudas imagenológicas, de las cuales se ha hecho mención anteriormente, en las que se puede considerar a la resonancia magnética (RM) como una importante contribuyente a la detección de compromiso vascular en caso de NT lo que permitirá excluir otras causas; además se destaca su alta sensibilidad, con tasas que van de 94-97\% (11). Adicionalmente, cuando se realiza la interpretación imagenológica es necesario tomar en consideración la existencia de variantes anatómicas existentes en sujetos sanos o no afectados por NT (12).

Tabla 1. Criterios Diagnósticos de Neuralgia del Trigémino.

\begin{tabular}{|c|c|}
\hline Localización: & Dermatoma trigeminal (generalmente unilateral) \\
\hline Radiación: & Hacia el áea trigeminal y otros dermatomas \\
\hline Características: & Tipo agudo, percepción de descargas de tipo eléctrico \\
\hline Intensidad: & $\begin{array}{l}\text { Moderado a severo (desencadenante de inestabilidad emocio- } \\
\text { nal hasta niveles de suicidio) }\end{array}$ \\
\hline \multirow{2}{*}{ Duración: } & 1. Múltiples episodios de dolor en el día. \\
\hline & 2. Duración de varios minutos por cada episodio de dolor. \\
\hline Frecuencia: & Alternancia entre episodios dolorosos y no dolorosos. \\
\hline $\begin{array}{l}\text { Factores } \\
\text { desencadenantes: }\end{array}$ & Presión leve \\
\hline Alivio del dolor: & Fármacos anti neurálgicos \\
\hline Factores asociados: & Puntos gatillos, pérdida de peso \\
\hline
\end{tabular}

Fuente: tomado de International Headache Society (IHS) (4) 


\section{¿Qué relación existe entre el odontólogo, la consulta odontológica y los pacientes con NT?}

Para responder a este interrogante se puede hacer referencia inicialmente a los procedimientos odontológicos que han sido asociados con lesiones al nervio trigémino, esto debido a que en ocasiones el paciente puede acudir a consulta con el odontólogo, por dolor inducido precisamente durante un tratamiento en sus dientes o en tejidos circundantes de la cavidad bucal. Algunos de los procedimientos que han sido relacionados con respuestas neurálgicas en el $\vee$ par craneal son las extracciones dentales, bloqueo anestésico, realización de tratamientos endodónticos y colocación de implantes (13).

Para el caso de la exodoncia quirúrgica, algunas de las manifestaciones clínicas que se pueden presentar son dolor, inflamación y trismo, siendo el dolor el más frecuente de los tres signos y síntomas anteriormente referidos. Así por ejemplo, se ha señalado que tras la exodoncia quirúrgica de un tercer molar inferior, el dolor es más severo durante las primeras 12 horas pero al existir una prolongación del mismo a más de 72 horas, se sugiere la probabilidad de haber desencadenado NT, ante lo cual es posible mencionar el estudio realizado por Haug $\mathrm{RH}$, Perrott DH, Gonzalez ML, Talwar RM, en Estados Unidos durante 2005, el cual demostró, tras realizar las extracciones de 8333 terceros molares, la presencia de complicaciones posquirúrgicas en las que $0,2 \%$ se asocia con NT (14), cifra que, aunque baja, constituye un aporte a la evidencia de que tras el procedimiento quirúrgico puede inducirse este trastorno neuropático. Por lo anterior, se sugiere como importante criterio clínico conjugar los antecedentes odontológicos de extracciones dentales que se obtienen durante la anamnesis y las características clínicas referidas por el paciente con la particularidad de presencia de dolor que recidiva y con periodos de alternancia (15) (considerar los criterios clínicos definidos para el diagnóstico de NT en la tabla 1); además, los profesionales de odontología deben afianzar constantemente su conocimiento clínico y radiográfico al momento de realizar los abordajes quirúrgicos para generar el menor trauma posible y disminuir la probabilidad de lesionar terminaciones del $\mathrm{V}$ par craneal.

Otro aspecto muy polémico es el concerniente al bloqueo anestésico, algunos estudios como el de Renton et al. (16) en Inglaterra, realizado en un total de 33 pacientes, halló la presencia de lesión nerviosa tipo NT, a consecuencia de bloqueo de los nervios lingual y alveolar inferior tras la aplicación de anestesia local. En algunos casos, se destaca como antecedente de NT el recibir múltiples inyecciones de manera inapropiada sobre el nervio (16). Otros estudios se enfocan en la parestesia del nervio trigémino, por bloqueo del nervio alveolar inferior, tal es el caso del estudio realizado 
por Van der Sleen J, Jaspers G, de Lange J (2015) en Países Bajos (17), en 1000 pacientes sometidos a cirugía oral, de los cuales 5 reportaron parestesia por bloqueo anestésico del nervio alveolar inferior y 6 pacientes por bloqueo del nervio lingual, las anteriores aunque cifras, no muy significativas, son orientadoras respecto a las repercusiones que puede tener una acción tan rutinaria en la consulta odontológica como lo es la colocación de anestesia. Por esto, se recomienda que el odontólogo tenga en cuenta los criterios anatómicos referentes a las estructuras sobre las que se va a aplicar la sustancia anestésica, a fin de prevenir las múltiples punciones o reducir la probabilidad de realizar punciones en zonas inadecuadas con la consiguiente inducción de neuropatías.

Respecto a los tratamientos endodónticos, la existencia de lesiones periapicales podría considerarse como factor de riesgo para NT, lo anterior podría explicarse por sobre-instrumentación de dientes próximos al canal del nervio alveolar inferior o por extravasación de sustancias citotóxicas o de material de relleno hacia tejidos periapicales, lo cual induce procesos inflamatorios capaces de generar compresión a ramas nerviosas. Para soportar un poco la anterior consideración, puede tenerse en cuenta lo reportado por Devine et al. (18) en Londres, a partir de una muestra de 352 pacientes con NT, donde se presentó que en un 6,2%, la presencia de NT se asoció con presencia de lesiones periapicales, las cuales comprometían a los primeros molares mandibulares en la mitad de los casos. Es por esto que resulta indispensable un correcto diagnóstico y tratamiento endodóntico por parte del especialista u odontólogo general, ya que existe la posibilidad ante determinadas condiciones que parecerían favorecer o aumentar el riesgo para desencadenar NT.

En lo concerniente a la colocación de implantes dentales, procedimiento que ha cobrado mucho auge en la actualidad, existe un reporte de aumento en la incidencia de lesión al nervio después de cirugía dental, incluso, se ha mencionado la existencia de una neuropatía trigeminal traumática, propuesta para situaciones en las que existe dolor neuropático que ocurre dentro de los tres meses de realizados los procedimientos. Este dolor neuropático en el maxilar inferior puede derivar sin evidencia de intrusión directa del implante hacia el canal alveolar inferior y puede obedecer al caprichoso y variable trayecto que puede tener el nervio en el canal, por lo anterior se sugiere una zona de seguridad de 2 milímetros por encima del canal alveolar inferior a fin de disminuir el riesgo de lesiones sobre el nervio (19). En un estudio realizado por Renton et al., (20) en 2012, sobre un total de 287 pacientes con lesiones al nervio trigeminal, se evidencia que 30 sujetos tenían neuropatía asociada a la colocación de implantes y que se presentaban alteraciones funcionales en actividades tales como besar, socializar, hablar, beber, comer, cepillado dental entre 
otros, destacando la importancia de informar a los pacientes respecto a la existencia de probabilidad de lesión al nervio, pues los síntomas resultaron estar agravados por falta de consentimiento informado, que sólo se obtuvo de 30 \% de los paciente (20). Por esta situación, en la dinámica de rehabilitar al paciente con implantes, se requiere una ardua preparación académica para los odontólogos, pues es otro de los procedimientos que, de no realizarse con los debidos cuidados, puede propiciar este tipo de respuestas neuropáticas.

Una vez se logra exponer la importancia de considerar algunos procedimientos odontológicos, como posibles inductores de la respuesta neuropática, es importante reflexionar un poco respecto a las situaciones de diagnóstico de NT que tienen lugar en la consulta odontológica, pues existe evidencia de estudios, en los cuales los participantes informan la existencia de retraso en el diagnóstico exacto, quizás esta consideración se deba a falta de formación y experticia en la temática que hace que los odontólogos a veces no posean un concepto claro. Lo anterior ya se ha descrito previamente, Aggarwal et al., (21) en 2011, reportan que la mayoría de los participantes con diagnóstico de NT se presentó a consulta odontológica y que se evidenció marcada brecha de conocimiento en dolor orofacial crónico, tanto en odontólogos generales como en odontólogos especialistas. Incluso en estudios como el de von Eckardstein et al., (22) en 2015, se concluyó la existencia de un alto porcentaje de pacientes que, en caso de NT, primero consultan a su odontólogo y reciben tratamiento dental posiblemente injustificado por lo que se puede considerar la existencia de discrepancias durante el diagnóstico. Lo anterior coincide con lo expuesto por Perić et al. (23), tras realizar un estudio en 116 pacientes referidos por los odontólogos de atención primaria, en el que se reporta que en 33,6 \% de los casos los diagnósticos son erróneos.

Finalmente, es posible destacar otra condición clínica que involucra el sistema estomatognático y que aunque no suele ser motivo de consulta frecuente para el odontólogo, implica un importante desafío para documentarse y mantenerse actualizado contribuyendo a su oportuno diagnóstico y tratamiento, esta condición dolorosa corresponde a disfunción o trastorno temporomandibular (24), el cual tiene similitudes con el dolor ocasionado por neuralgia del trigémino, comportándose así como un diagnóstico diferencial (25).

Por todo lo anterior, se puede tomar en consideración la urgente necesidad de afianzar y mejorar la formación de los odontólogos respecto a NT para contribuir en su oportuno diagnóstico. 


\section{Tratamiento para NT}

Aunque el odontólogo no es el protagonista principal en la instauración de tratamiento en caso de NT, pues sus funciones obedecen al diagnóstico inicial, referencia y colaboración en el mantenimiento integral de la salud bucal del paciente, debe reconocer las opciones terapéuticas farmacológicas y quirúrgicas para NT (tabla 2). En este aspecto es importante destacar que las opciones de manejo quirúrgico se encuentran disponibles cuando los medicamentos fracasan o sus efectos secundarios son demasiado graves $(26,27)$. En lo concerniente a la terapia farmacológica, los medicamentos para controlar la sintomatología dolorosa se clasifican en una primera línea conformada por los medicamentos Carbazepina y Oxcarbazepina, segunda línea representada por Lamotrigina y Baclofen, tercera línea conformada por Levetiracetam, Gapabentina, Pregabalina y Topiramato $(28,29)$. Otra alternativa a considerar, corresponde al uso de vitaminas del complejo B (específicamente tiamina, piridoxina y cobalamina) que, si bien es cierto, no es considerado como un tratamiento de primera elección, sino como coadyuvante o en caso de existir su défıcit, se ha documentado en pruebas de manera experimental y clínica que la vitamina B1 y B12 poseen efectos farmacológicos en la conducción axonal (como la analgesia) y excitabilidad $(4,30,31)$.

Tabla 2. Alternativas tratamiento para NT.

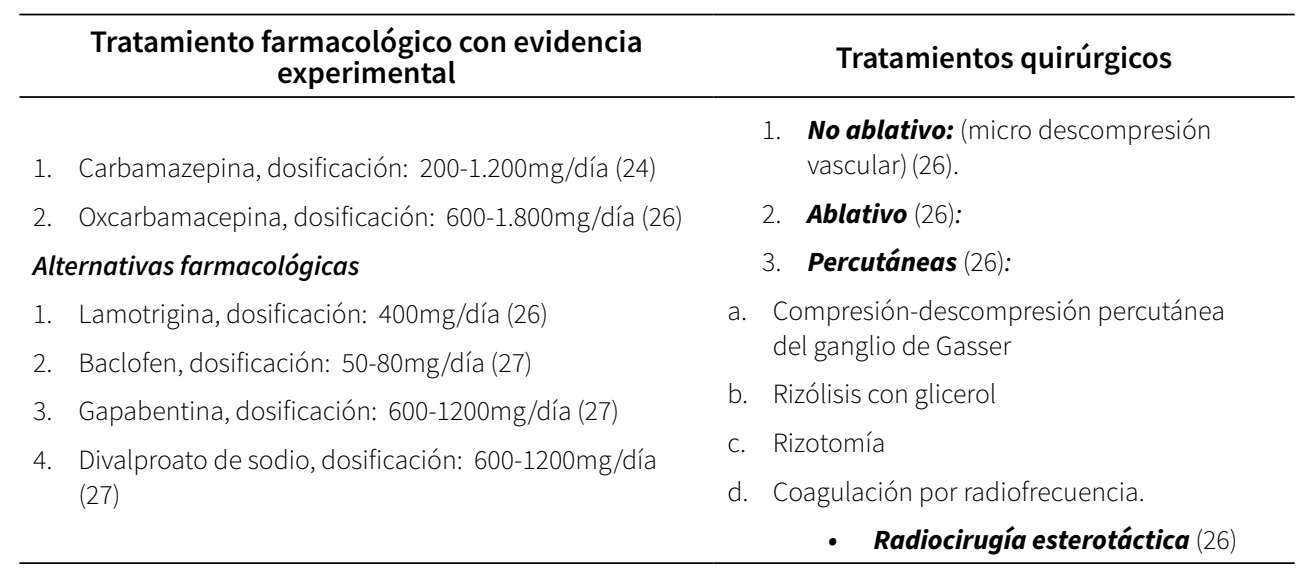

Fuente: elaboración propia 


\section{Conclusión}

La NT es una alteración que repercute sobre la calidad de vida del paciente, al generar sintomatologías constituidas principalmente por los episodios dolorosos e incapacitantes. Es necesario considerar el importante rol que puede asumir el odontólogo y que involucra inicialmente el reconocimiento de que existen algunas condiciones durante los procedimientos en la consulta odontológica que pueden desencadenar NT. Adicionalmente, se requiere de sus competencias como clínico para la identificación de síntomas sugestivos de esta alteración, lo que a su vez permitirá realizar el correcto y oportuno diagnóstico, así como evaluar la posibilidad de remitir con los especialistas específicos en el área de atención de neuropatías.

\section{Referencias}

1. Chávez O, Martínez L, Cazares J, Martínez M, Roldan E. Anatomía de los nervios craneales con resonancia magnética de 3.0 Tesla: una revisión práctica para clínicos. Gaceta Médica de México. 2011; 147: 526-37.

2. Reddy GD, Viswanathan A. Trigeminal and glossopharyngeal neuralgia. Neurol Clin. 2014; 32: 539-52. doi: 10.1155/2017/7438326

3. Da Silva OT, de Almeida CC, Iglesio RF, de Navarro JM, Teixeira MJ, Duarte KP. Surgical variation of microvascular decompression for trigeminal neuralgia: A technical note and anatomical study. Surgical Neurology International. 2016; 7(Suppl 21): S571-S576. doi: 10.4103/2152-7806.188916.

4. Chumpitaz V. Actualización de Criterios Diagnósticos y Tratamiento de la Neuralgia del Trigémino. Odontol. Sanmarquina 2013; 16(1): 44-48.

5. Cruccu G, Finnerup NB, Jensen TS, Scholz J, Sindou M, Svensson P, et al. Trigeminal neuralgia: New classification and diagnostic grading for practice and research. Neurology. 2016; 87(2): 220-228. doi: 10.1212/WNL.0000000000002840.

6. Alcántara A, Sánchez Cl. Actualización en el manejo de la neuralgia del trigémino. Semergen. 2016; 42(4): 244-253 
7. Fernández Rodríguez B, Morollón N, Guerrero P, Tabernero C, et al. Neuralgia del trigémino clásica familiar. Neurologia. 2017 Mar 24; pii: S0213-4853(17)30019-1. doi: 10.1016/j. nrl.2016.12.004.

8. Boto G. Neuralgia del trigémino. Neurocirugía. 2010; 21: 361-372.

9. D’Amico A, Russo C, Ugga L, Mazio F, Capone E, D’Arco F, et al. Can pontine trigeminal T2-hyperintensity suggest herpetic etiology of trigeminal neuralgia? Quant Imaging Med Surg. 2016; 6(5): 490-495. doi: 10.21037/qims.2016.01.07.

10. Sabalys G, Juodzbalys G, Wang HL. Aetiology and Pathogenesis of Trigeminal Neuralgia: a Comprehensive Review. Journal of Oral \& Maxillofacial Research. 2012; 3(4): e2. doi: 10.5037/ jomr.2012.3402.

11. Hughes MA, Frederickson AM, Branstetter BF, Zhu X, Sekula RF Jr. MRI of the Trigeminal Nerve in Patients With Trigeminal Neuralgia Secondary to vascularCompression. AJR Am J Roentgenol. 2016; 206(3): 595-600. doi: 10.2214/AJR.14.14156.

12. Lutz J, Linn J, Mehrkens J, Thon N, Stahl R, Seelos K. Trigeminal Neuralgia due to Neurovascular Compression: High-Spatial-Resolution DiffusionTensor Imaging Reveals Microstructural Neural Changes. Radiology. 2011; 258(2): 524-530. doi: 10.1148/radiol.10100477.

13. Hillerup S. Iatrogenic injury to oral branches of the trigeminal nerve: records of 449 cases. Clin Oral Investig. 2007; 11(2): 133-142. doi: 10.1007/s00784-006-0089-5.

14. Haug RH, Perrott DH, Gonzalez ML, Talwar RM. The American Association of Oral and Maxillofacial Surgeons Age-Related Third Molar Study. J Oral Maxillofac Surg. 2005 Aug; 63(8): 1106-14. doi: 10.1016/j.joms.2005.04.022.

15. Allsop MJ, Twiddy M, Grant H, Czoski-Murray C, Mon-Williams M, Mushtaq, F et al. Diagnosis, medication, and surgical management for patients with trigeminal neuralgia: a qualitative study. Acta Neurochirurgica. 2015; 157(11): 1925-1933. doi: 10.1007/s00701-015-2515-4.

16. Renton T, Adey-Viscuso D, Meechan JG, Yilmaz Z. Trigeminal nerve injuries in relation to the local anaesthesia in mandibular injections. Br Dent J. 2010 Nov; 209(9): E15. doi: 10.1038/ sj.bdj.2010.978.

17. Van der Sleen J, Jaspers G, de Lange J. Trigeminal nerve paraesthesia from inferior alveolar nerve blocks: incidence and course. Oral Surgery. 2015; 8(4): 217-220. doi: 10.1111/ors.12157. 
18. Devine M, Yilmaz Z, Hirani M, Renton T. A case series of trigeminal nerve injuries caused by periapical lesions of mandibular teeth. Br Dent J. 2017;222 (6): 447-455. doi: 10.1038/ sj.bdj.2017.268.

19. Delcanho R, Moncada E. Persistent pain after dental implant placement: a case of implant-related nerve injury. J Am Dent Assoc. 2014; 145(12): 1268-71.

20. Renton T, Dawood A, Shah A, Searson L, Yilmaz Z. Post-implant neuropathy of the trigeminal nerve. A case series. Br Dent J. 2012 Jun 8; 212(11): E17. doi: 10.1038/sj.bdj.2012.497.

21. Aggarwal VR, Joughin A, Zakrzewska JM, Crawford FJ, Tickle M. Dentists' and specialists' knowledge of chronic orofacial pain: results from a continuing professional development survey. Prim Dent Care. 2011; 18(1): 41-44. doi: 10.1308/135576111794065838.

22. von Eckardstein KL, Keil M, Rohde V. Unnecessary dental procedures as a consequence of trigeminal neuralgia. Neurosurg Rev. 2015 Apr; 38(2): 355-60. doi: 10.1007/s10143-014-0591-1.

23. Perić B, Zore IF, Vidaković B, Jokić D, Cabov T, Sarac H. Trigeminal neuralgia--case and treatment analysis at the Department of Oral Surgery of the University Hospital Dubrava. Coll Antropol. 2014 Jun; 38(2): 685-9.

24. Tirado-Amador LR. Trastornos temporomandibulares: algunas consideraciones de su etiología y diagnóstico. Rev Nac Odontol. 2015; 11(20): 83-93. doi: http://dx.doi.org/10.16925/ od.v11i20.748

25. Pihut M, Szuta M, Ferendiuk E, Zeńczak-Więckiewicz D. Differential Diagnostics of Pain in the Course of Trigeminal Neuralgia and Temporomandibular Joint Dysfunction. BioMed Research International. 2014. doi: 10.1155/2014/563786.

26. Bescòs A, Pascual V, Escosa M, Málaga X. Tratamiento de la neuralgia del trigémino: actualización y perspectivas futuras de las técnicas percutáneas. Revista Neurología. 2015; 61(3): 114-124.

27. García M, Sánchez J, Tenopala S. Neuralgia del trigémino. Revista Anales médicos. 2012; 57(1): 39-47.

28. Cruccu G, Gronseth G, Alksne J, Argoff C, Brainin M, Burchiel K, Nurmikko T, Zakrzewska JM, American Academy of Neurology Society., European Federation of Neurological Society. Eur J Neurol. 2008 Oct; 15(10): 1013-28. doi: 10.1111/j.1468-1331.2008.02185.x. 
29. Al-Quliti KW. Update on neuropathic pain treatment for trigeminal neuralgia: The pharmacological and surgical options. Neurosciences (Riyadh). 2015 Apr; 20(2): 107-114. doi: 10.17712/ nsj.2015.2.20140501.

30. Torres y Gutiérrez Rubio A. Sobre el efecto analgésico de las vitaminas del complejo B, a 50 años de la primera combinación fija de tiamina, piridoxina y cianocobalamina. Med Int Mex. 2012; 28(5): 473-482.

31. Aggarwal A, Wood I. Low Vitamin B12 Syndrome in Trigeminal Neuralgia. J Pain Relief. 2012; 1: 109. doi: 10.4172/2167-0846.1000109. 